Portland State University

PDXScholar

\title{
A Daily Examination of Anger and Alcohol Use Among Post-9/11 Veterans
}

James David Lee

Portland State University

Follow this and additional works at: https://pdxscholar.library.pdx.edu/open_access_etds

Part of the Military and Veterans Studies Commons, and the Psychology Commons Let us know how access to this document benefits you.

\section{Recommended Citation}

Lee, James David, "A Daily Examination of Anger and Alcohol Use Among Post-9/11 Veterans" (2020). Dissertations and Theses. Paper 5587.

https://doi.org/10.15760/etd.7459

This Thesis is brought to you for free and open access. It has been accepted for inclusion in Dissertations and Theses by an authorized administrator of PDXScholar. Please contact us if we can make this document more accessible: pdxscholar@pdx.edu. 
A Daily Examination of Anger and Alcohol Use among Post-9/11 Veterans

by

James David Lee

A thesis submitted in partial fulfillment of the requirements for the degree of

Master of Science

in

Psychology

Thesis Committee:

Cynthia D. Mohr, Chair

Todd E. Bodner

Leslie B. Hammer

Portland State University

2020 
C2020 James David Lee 


\begin{abstract}
Anger problems have been commonly reported among military service-connected individuals. Current estimates of self-reported anger issues among post-9/11 veterans are around 57\% (Sayer et al., 2010). Alarmingly, it's been reported that anger does not decrease over the course of the post-deployment period (Bliese et al., 2007; Heesink et al., 2015); left unmanaged, it has been associated with a higher risk for suicide (Kessler et al., 2014), post-traumatic stress (Forbes et al., 2008), and hazardous alcohol use (Steele \& Fogarty, 2017). Heavy drinking within military-connected samples has also been a welldocumented phenomenon. Recent findings suggest that service-connected individuals may cope with adversity and regulate emotions through alcohol use, which may put them at risk for developing alcohol use disorders (e.g., Miller, Pedersen, \& Marshall, 2017). While some evidence has observed a positive link between anger and alcohol use (Jakupcak et al., 2007), the temporal relationship between anger and drinking is not fully understood. Little research has investigated intraindividual patterns of anger and drinking behaviors from a daily perspective, which is an important gap for this population who frequently report problems associated with both constructs. Therefore, the current study aimed to elucidate the relationship between daily anger, trait anger, and drinking behaviors from a daily diary perspective. Participants were comprised of a subsample of employed post-9/11 veterans $(n=101)$ who were recruited as part of the Study for Employment Retention of Veterans (SERVe) and were classified as regular-shift workers who consumed alcohol. Data were analyzed using within-subject multilevel regression models over time. Findings of the study indicated that the daily anger of veterans was
\end{abstract}


negatively related to the number of evening drinks they consumed-especially among those with lower levels of trait anger. This study provides insight into the intrapersonal drinking behaviors of veterans in the workplace, a population rarely studied. 


\section{Acknowledgments}

I would like to extend my deepest gratitude to my advisor, Dr. Cynthia Mohr, who has provided me with unwavering support and guidance throughout my graduate school career while modeling what it means to be an inquisitive investigator. I would also like to thank Drs. Leslie Hammer and Todd Bodner, for providing valuable opportunities, insights, and expertise throughout this thesis project and during my time as a research assistant, you have been instrumental to my growth as a researcher. To my colleagues, friends, and family, you inspire me to stay the course when times get tough and supply no shortage of entertainment and laughter. Finally, to my loving wife, Melissa. Your patience and optimism inspire me to be ambitious. From the military to graduate school, I've been blessed to have you by my side each step of the way.

The U.S. Army Medical Research Acquisition Activity, 820 Chandler Street, Fort Detrick MD 21702-5014 is the awarding and administering acquisition office. This work was supported by the Office of the Assistant Secretary of Defense for Health Affairs, through the USAMRMC Broad Agency Announcement under Award W81XWH-13-2-0020. Opinions, interpretations, conclusions and recommendations are those of the author and are not necessarily endorsed by the Department of Defense. 


\section{Table of Contents}

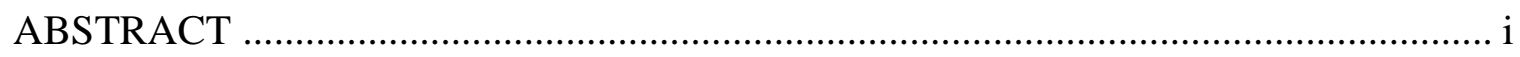

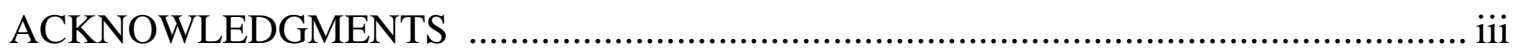

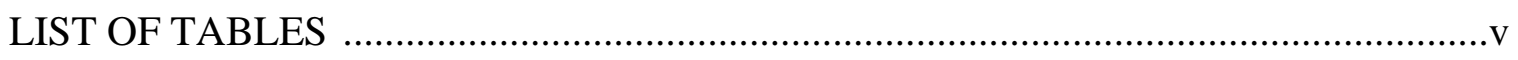

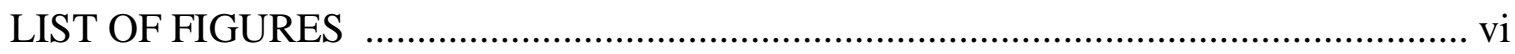

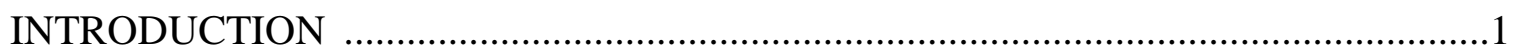

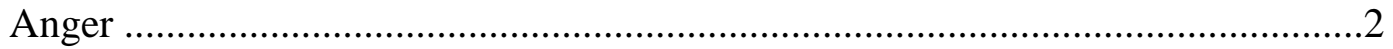

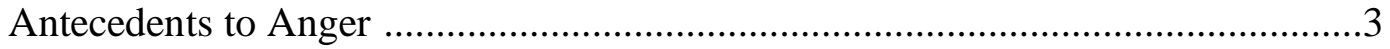

Outcomes of Anger .................................................................................. 7

Alcohol Use ....................................................................................... 9

Theoretical Links between Anger and Alcohol Use ......................................... 10

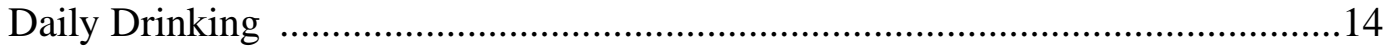

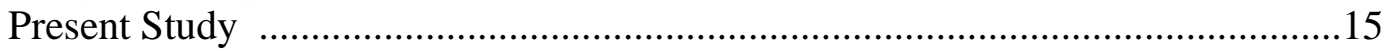

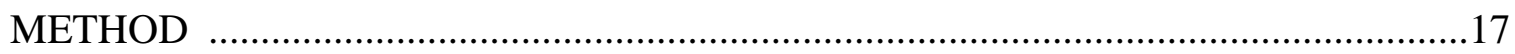

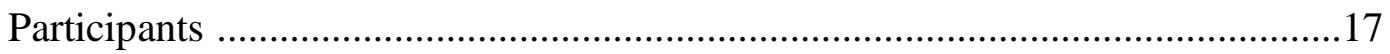

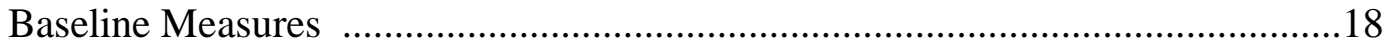

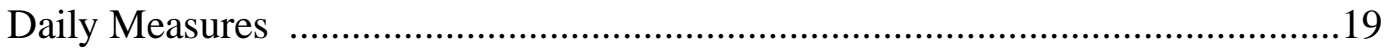

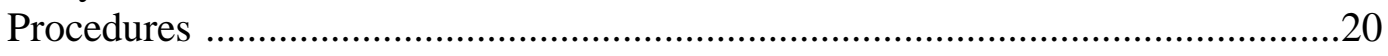

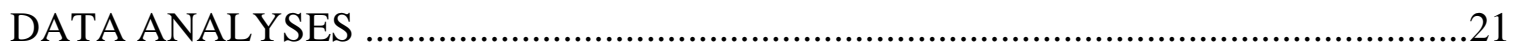

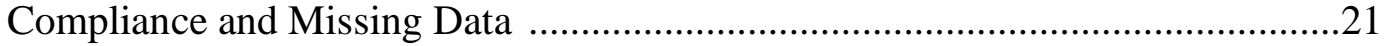

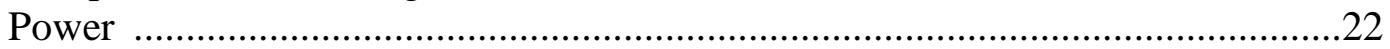

Variable Management .............................................................................2

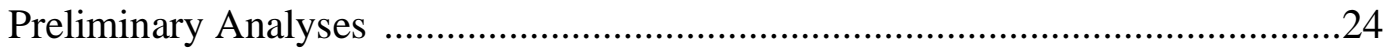

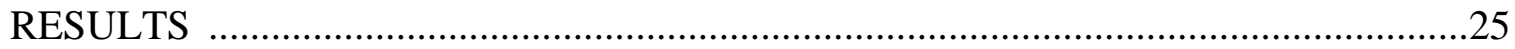

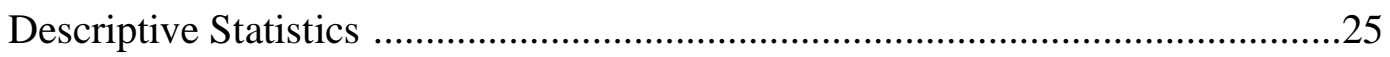

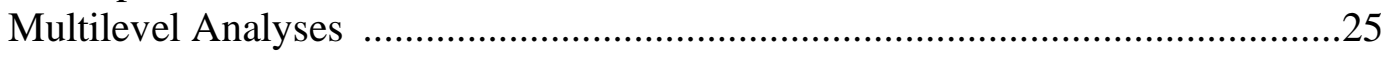

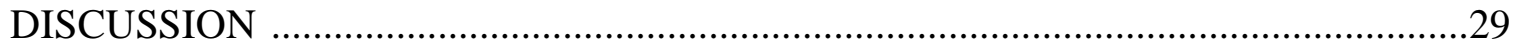

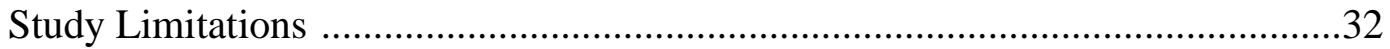

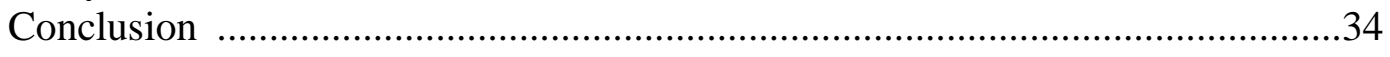

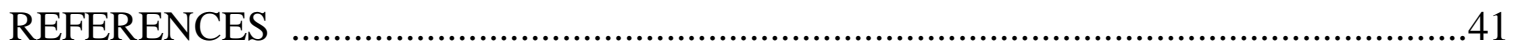

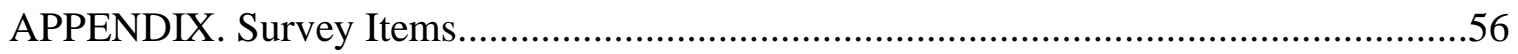




\section{List of Tables}

TABLE 1. Sociodemographic \& Military Characteristics ……………………….............36

TABLE 2. Descriptives and Inter-Correlations Among Study Variables .........................37

TABLE 3. Multilevel Regression Results for Study Variables ………….........................38 


\section{List of Figures}

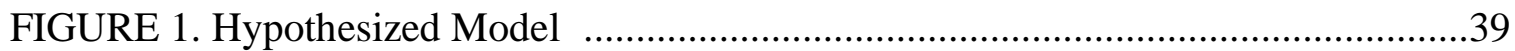

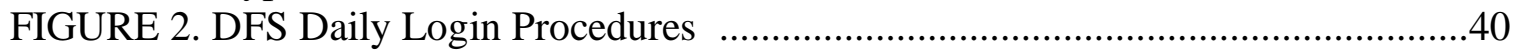




\section{INTRODUCTION}

The events of 9/11 led the United States to engage in multiple military operations in the Middle East including Operation Enduring Freedom, Operation Iraqi Freedom, and Operation New Dawn. Colloquially known as “America's Longest War”, such conflicts have resulted in over 2.8 million service members being deployed to support the war efforts (Tanielian, Batka, \& Meredith; 2017). The austere nature of the combat theater may subject military personnel to traumatic experiences including being ambushed, killing the enemy, or witnessing fellow comrades being injured or killed (Hoge et al., 2004). Unsurprisingly, these traumatic stressors have taken a toll on American warfighters as many return from combat with an increased risk for substance use, suicidal ideation, and mental health problems (RAND, 2019). Other findings related to deployment experiences among veterans include an increased risk for sudden anger outbursts and alcohol dependence (Tanielian, Batka, \& Meredith, 2017). While many studies have focused on the substance use of military-connected individuals, research on veteran anger has been scant. Yet, available data reveals that $57 \%$ of post-9/11 veterans experience anger-related issues (Sayer et al., 2010), which may put them at risk for committing acts of aggression (Jakupcak et al., 2007).

These traumatic environmental factors may explain why service-connected individuals have different levels of anger when compared to the civilian population. Within the military context, the expression of anger, aggression, and use of force are conducive to survival and quell feelings of helplessness during combat (Forbes et al., 2008; Chemtob, Novaco, Hamada, Gross, \& Smith, 1997). However, multiple studies 
have found that anger levels do not decrease following post deployment (Bliese et al., 2007; Heesink et al., 2015) and left unmanaged, anger may result in higher risks for suicide, posttraumatic stress disorder (PTSD), and hazardous alcohol use (Kessler et al., 2014; Forbes et al., 2008; Steele \& Fogarty, 2017).

The primary goal of this thesis is to examine the relationship between anger and alcohol consumption among veterans. In the following document, I provide a review of the literature surrounding service-connected individuals while proposing a hypothesized relationship between anger and drinking behaviors from a daily perspective. Specifically, the first section will cover the experience of anger while defining its many forms as well as their antecedents and outcomes. Next, anger will be addressed in addition to how it relates to the military context and drinking behaviors. The third section will describe facets that relate to alcohol use including motivations and self-control, while considering daily process methodology. Finally, the last section will introduce the hypothesized within-person relationships that are thought to occur among veterans and will provide further details regarding the design of the study.

\section{Anger}

Anger is a basic human emotion and has been an elusive construct to define as its multidimensional nature consists of affective, cognitive, behavioral, and physiological components, which ultimately influence how anger is experienced and expressed (Spielberger, 1983). Spielberger's (1983) State-Trait Theory of anger delineates between two main dimensions of anger, i.e., state anger and trait anger, with state anger being defined as an "emotional state that consists of feelings that vary in intensity, from mild 
irritation or annoyance to intense fury and rage" (p.162). In essence, state anger centers around the temporary state (episode) of anger as it relates to the immediate situation, whereas trait anger has been conceptualized as an individual's propensity to experience anger in terms of frequency, intensity, and duration. Trait anger is a common antecedent to state anger and individuals with high levels of trait anger tend to interpret situations as being more hostile and have difficulty controlling their hostile-related thoughts and feelings (Veenstra, Bushman, \& Koole, 2018). The way anger is expressed has been stratified by Spielberger (1988) through his State-Trait Anger Expression Inventory which includes the following dimensions: anger-in, anger-out, and anger control. Angerin refers to the extent to which an individual directs their anger internally (anger suppression), anger-out is characterized by the extent in which individuals express their anger outward towards people or objects, and anger-control is the extent in which individuals have control over their anger expressions. While the construct of anger has been described by its affective dimension and consists of an emotional experience (Cox \& Harrison, 2008), its behavioral manifestation has generally been regarded as aggression and is defined as "any behavior directed toward another individual that is carried out with the immediate intent to cause harm" (Anderson \& Bushman, 2002, p. 28)."

\section{Antecedents of Anger}

In an attempt to elucidate what leads to anger, it may be beneficial to examine antecedents to anger while considering psychological, social, and cultural perspectives. Before delving into each dimension, it is important to note that in terms of gender, much 
of the literature suggests that men and women experience anger at similar intensities, express it in similar ways, and are angered by similar stimuli (Averill, 1982). This finding is further supported by research suggesting that the relationship between anger and PTSD severity is similar across genders (Worthen et al., 2014). Finally, Gianakos (2002) examined anger-related issues in the workplace and reported no gender differences in regards to the type or number of issues that elicited anger nor the methods used to cope with anger.

Starting from a psychological lens, some evidence suggests that the experience of anger is contingent upon an individual's appraisal of an event. Specifically, Deffenbacher (1994, p. 243) posited that anger occurs when an individual perceives that a violation has occurred that impacts their domain or their way of living. Stated alternatively, when one's expectations or goals are interrupted or broken, anger is likely to result, especially if the stimuli were deemed to be unjustified and/or intentional. These assertions are supported by theoretical models that describe how anger leads to aggression. In their seminal publication, Dollard and colleagues (1939) elucidated how frustration led to aggression when one's goals were blocked or interrupted; Berkowitz (1989) advanced the hypothesis by contending that frustrations lead to aggression to the degree to which they generate negative affect, thereby capturing the mediational role of anger as it relates to the relationship between frustration and aggression. In considering social influences of anger, Bandura's social learning theory of aggression (1978) describes how aggression is learned by either observing others or through rewards for perpetuating aggression. Within the context of the warfighter, service members are encouraged to react with anger and/or 
aggression in order to ensure the safety of themselves or others-such behavior is also critical in applications of national defense.

The rules and tolerance of anger expression may also vary as a function of culture. For example, the "culture of honor" in Southern U.S. states carries a reputation of inflicting aggression and violence in order to preserve one's reputation and rights, discourage potential threats to one's family, and to minimize theft (Nisbett \& Cohen, 1996). Not surprisingly, researchers have found that U.S. presidents who were raised in southern cultures were "twice as likely to use force, experience disputes that last on average twice as long, and are three times as likely to achieve victory" in regards to international conflicts (Dafoe \& Caughey, 2016, p. 371). Tavris (1989) sheds light on the varying implicit and explicit rules of anger within respective cultures. In essence, ingroup members learn which anger responses are appropriate within their culture and strategies are implemented to curb inappropriate anger. Furthermore, Tavris reports how cultures dictate when and under what conditions, the expression of anger and rage are acceptable. When applied to a combat setting, it becomes clear that service members are given the "green light" to direct anger, rage, and fury towards enemy combatants as long as their actions do not violate the mission's rules of engagement and/or military law.

Within the military context, experiencing anger may be useful as it activates aggression, which in turn, may give impetus to attack and survival behaviors as well as quell feelings of helplessness in combat situations (Chemtob, Novaco, Hamada, Gross, \& Smith, 1997). Similarly, Reyes and Hicklin (2005) noted that there are precursors associated with military service that may engender the experience of anger. Specifically, 
the authors stated that soldiers who are deployed within a combat zone experience multiple stressors such as lack of physical comforts, environmental hazards, and threats from opposing forces, which may contribute to anger, frustration and rage. Similarly, research has found that combat experiences influence veteran's anger levels, evidenced by the fact that $47 \%$ of veterans with deployment experience suffer from sudden outbursts of anger (Tanielian, Batka, \& Meredith, 2017).

Self-selection related factors have been briefly considered as the prospect of military service has been shown to attract individuals from low socioeconomic backgrounds, those with lower social-connectedness, and individuals with a history of adolescent fighting (Elder et al., 2010), which may presumably contribute to trait anger or aggression. However, the military socialization process also entails cultivating discipline and self-control and research has yet to identify anger or aggression trajectories pre-, during, and post- military service.

Unfortunately, limited attempts have been made to shed light on the association between personality traits and the decision to enlist in the Armed Forces. Jackson, Thoemmes, Jonkmann, Lüdtke, and Trautwein (2012) were one of the first to examine personality traits and military training in a longitudinal sample of German males and found that adolescents who enlisted in military service had significantly lower levels of agreeableness, neuroticism, and openness to experience during high school. After controlling for baseline levels of agreeableness, Jackson and colleagues (2012) conducted a socialization analysis and found that agreeableness was the only personality trait to change as a result of military training. Furthermore, the authors make the argument that 
low levels of agreeableness may be conducive in military contexts considering that individuals with high levels of agreeableness are a) less likely to react with aggression and b) have slower reaction times when prompted with aggression cues (Meier, Robinson, \& Wilkowski, 2006). Whether these findings generalize to U.S. armed forces has yet to be determined, but it does lend credence to the argument that aggression is adaptive within military contexts. Nonetheless, it is apparent that military culture shares an interesting relationship with anger and aggression. On one hand, the expression of anger and aggression is conducive to survival in combat application and is instrumental in defending a nation. Conversely, the military values personal characteristics of order, self-control, and discipline among its personnel (Linkh \& Sonnek, 2003). In terms of trait anger, military culture and training have been found to be antecedents to trait anger because warfighters are trained to respond to threat or vulnerability with anger, aggression, and use of force (Forbes et al., 2008).

\section{Outcomes of Anger}

Anger can lead to both positive and negative outcomes, and findings of anger expression have yielded mixed outcomes. Positive outcomes of anger include the ability to express emotions in a non-hostile manner which may assist in identifying problems and redressing issues for all individuals involved (Averill, 1982). In some settings, anger has been shown to improve work performance through problem solving or by serving as a motivator (Glomb \& Hulin, 1997). Alternatively, organizational scholars have asserted that trait anger plays a fundamental role in the occurrence of workplace aggression (Veenstra, Bushman, \& Koole, 2018) as employees with more trait anger report chronic 
workplace anger (Booth \& Mann, 2005). Anger has also been shown to degrade communication accuracy within the workplace (Rothman \& Magee, 2016) and lead to incivility (Andersson \& Pearson, 1999). Similarly, in their investigation between perceived organizational support, anger, and workplace outcomes, O'Neill, Vandenberg, Dejoy, and Wilson (2009) found the relationship between low perceived organizational support and workplace accidents, turnover intentions, and absences to be partially mediated by anger. While most studies reporting on the outcomes of anger have utilized civilian samples, it is prudent to consider whether similar results emerge among military personnel given the stark differences between military and civilian culture. For example, Lindebaum, Jordan, and Morris (2016) found that when military leaders expressed anger, it motivated their subordinates to reflect on the precipitating events that lead to their leader's expression of anger, which was associated with increases in their subsequent performance. Generally, findings within the civilian literature have indicated that the experience of anger adversely affects individuals through reduced job satisfaction (Glomb, 2002) and coronary heart disease (Smith, Glazer, Ruiz, \& Gallo, 2004). In a sample of service-members, Wilk and colleagues (2015) found an association between trait anger and an increased likelihood of developing treatment, legal, and interpersonalrelated problems. While the aforementioned benefits of anger are suitable for combat, it may not be the case for service-members in garrison environments. For example, Adler, Brossart, and Toblin (2017) found that some soldiers thought positively about their anger and believed that it helped them fulfill their occupational roles. However, the authors found evidence suggesting that this line of thinking was associated with worsened mental 
health and alcohol problems. Similarly, screening service-connected individuals by their levels of respective trait anger may prove to be useful in applied and clinical settings by identifying service members who may be at increased risk for aggression and angerrelated issues or alternatively, assist in identifying those who may be less likely to adhere to mental health treatments (Feeny, Zoellner, \& Foa, 2000).

\section{Alcohol Use}

It has been well established that service-connected individuals have high rates of alcohol use regardless of status (i.e., active duty, guard/reserves, veteran) and when compared to the civilian population, it has been estimated that service members, regardless of gender, are twice as likely to be heavy drinkers (Bray et al., 1991). Bray and colleagues (2010) found that $20 \%$ of military personnel engage in binge drinking at least once per week while Santiago and colleagues (2010) found that $27 \%$ of their combat veterans met criteria for alcohol misuse. In regards to studies carried out by Veterans Affairs (VA), Sayer and colleagues (2010) investigated reintegration problems among combat veterans seeking treatment at the VA and found that $38 \%$ of Iraq/Afghanistan veterans have an alcohol or drug use problem. Among post-9/11 veterans with deployment experiences, Tanielian, Batka, and Meredith (2017) reports that 15-44\% experience alcohol dependence. It is also important to note that little research has examined drinking rates among nonclinical veteran samples or veterans who do not utilize VA services. Though, in a previous analysis of the current study sample of veterans recruited from workplaces, Mohr, McCabe, Haverly, Hammer, and Carlson (2018) reported that nearly $24 \%$ met criteria for hazardous alcohol use. Interestingly, the 
majority of the workplace sample was comprised of veterans who had separated from the military after multiple years.

Robust associations have been found between anger, PTSD, and alcohol abuse (Deffenbacher \& DiGiuseppe, 2002; Hawthorne, Mouthann, Forbes, \& Novavo, 2006; Reyes \& Hicklin, 2005). Anger is also frequently found in cases of chronic alcohol abuse and PTSD (Castillo, Fallon, Baca, Conforti, \& Qualls, 2002), both of which are omnipresent issues that affect service-connected individuals. Within an active duty sample, Hoge and colleagues (2004) found that service members rates of alcohol misuse were significantly higher following deployment when compared to rates prior to deployment, which is congruous with reports indicating that $40 \%$ of service members experience increased drinking post-deployment (DMDC, 2010). Furthermore, Forbes and colleagues (2008) found anger to be a predictor of treatment outcomes among veterans with PTSD and is specifically determined by the extent in which alcohol comorbidity and a service member's fear of their anger is present. Moreover, the authors posit that the nature of military indoctrination entails training service members to respond to threat with aggression and lethal force. Thus, service members with alcohol problems may be more fearful of their anger being triggered due to the disinhibiting properties of alcohol, which in turn, may negatively affect their treatment outcomes. Thus, it is crucial to examine alcohol use while being mindful of the effects of anger.

\section{Theoretical Links Between Anger and Alcohol Use}

There are a variety of reasons to explain the high rates of alcohol use found among military personnel and veterans that span social and emotional perspectives. In 
terms of the former, it has been suggested that drinking culture within the military may glamorize excessive alcohol use (Browne et al., 2008) as service members spend much time together and live within close proximity to each other. These learned behaviors may also follow the service member as they transition into their veteran status. Of particular relevance to the present study, there are also motivational factors that may explain why service-connected individuals have higher rates of alcohol use. Much research has investigated drinking motivations; most notably, the seminal Tension Reduction Hypothesis (Conger, 1956) posited that individuals may be motivated to consume alcohol in order to reduce tension. Similarly, the self-medication model (Khantzian, 2003) has posited that individuals use alcohol to soothe psychological distress and has been used to describe comorbid conditions of alcohol misuse and PTSD among veterans (Langdon et al., 2016). Thus, service-connected individuals who drink to cope with stress may perpetuate a harmful cycle of behaviors which may lead to heavy drinking or alcohol use disorders. Cooper's (1994) four-factor model distinguished distinct drinking motives including coping, social, enhancement, and conformity motives. Thus, Cooper's model provides insight in determining whether individuals drink to socialize with others, enhance positive affect, cope with their negative emotions, or to conform to the group behaviors in order to reduce social pressures. Furthermore, Cooper, Frone, Russell, and Mudar (1995) articulated how individuals drink to regulate their emotions. Specifically, the authors asserted that individuals use alcohol to cope with negative emotions, which promote coping motivations or to enhance positive emotions via experience-enhancement motivations. These motivations were found to remain constant across age, gender, and 
racial groups. This assertion was supported by Mohr, McCabe, Haverly, Hammer, and Carlson (2018) who found that drinking-to-cope and enhancement-drinking motives were predictive of alcohol use among veterans, with drinking-to-cope motives being especially predictive of alcohol-related problems when controlling for psychological distress.

To further explore the link between posttraumatic stress symptoms (PTSS) and alcohol motivations among service-connected individuals, McCabe, Mohr, Hammer, and Carlson (2019) revealed indirect associations between posttraumatic stress symptoms and alcohol consumption through coping and enhancement drinking motives; This suggests that of the four previously-mentioned drinking motivations, coping and enhancement motives may be the most salient when examining drinking behaviors of combat-exposed veterans. Collectively, the tension reduction hypothesis and findings centered around enhancement and coping motives partially explain why veterans may develop alcohol problems. These perspectives explain how veterans who experience a preponderance of anger may engage in attempts to elevate their mood or manage their negative emotions through alcohol use.

The relationship between anger and alcohol use can also be understood from the perspective of the Strength Model of Self-Control (Baumeister, Vohs, \& Tice, 2007), which asserts that self-control is a finite resource that can become depleted through repeated exertions and result in adverse outcomes through the weakened ability to control one's impulses. For example, some experiments aimed at depleting self-control have led to overspending, overeating, and greater consumption of alcohol and nicotine than intended (Vohs \& Faber, 2007; Tangney, Baumeister, \& Boone, 2008; Christiansen, 
Cole, \& Field, 2012; Muraven, Collins, \& Nienhaus, 2002; Shmueli \& Prochaska, 2012). Berkowitz (2012) asserted that attempts to suppress negative feelings could backfire and result in extended thoughts of anger-especially when one's self-control abilities have been compromised by stressors, cognitive load, or time pressure. Similarly, Spector, Fox, and Domagalski (2006) hypothesized that anger leads to aggression by weakening the inhibitions involved in self-regulation. Congruously, Shorey, McNulty, Moore, and Stuart (2017) found evidence suggesting that trait anger moderated the relationship between alcohol use and physical aggression among university men. This moderating relationship was also found between heavy alcohol use and sexual aggression. Considering that anger rumination requires self-control coupled with the fact that self-control can be depleted, it becomes theoretically plausible that veterans with high levels of trait anger are facing many anger-provoking stimuli, which in turn, are attenuating their self-control; thus, opening the door to alcohol consumption in order to cope with their anger or stressors. In sum, multiple theoretical perspectives provide support for the proposed relationship between anger and alcohol consumption. Put simply, veterans may be drinking when angry to reduce tension or use alcohol as a means to self-medicate and attenuate other symptoms (e.g., posttraumatic stress). When considering the strength model of self-control, it becomes clear that veterans have a finite amount of self-control. As daily stressors arise, the veteran who struggles with controlling their anger in addition to drinking behaviors, may struggle with attempting to control both behaviors. These perspectives support the notion that more research is needed to examine the interplay 
between both constructs as they relate to the veteran and highlight the utility of observing the phenomenon through daily process methods.

\section{Daily Drinking}

Much of the research investigating anger and drinking behaviors has employed cross-sectional methods. However, to fully elucidate the relationship between moods and drinking behaviors among veterans, daily methodology should be considered. Research examining specific moods and drinking behaviors have shed insight into temporal associations at play which may ultimately influence drinking outcomes. Anger in particular, has been associated with higher levels of daily drinking within an adolescent sample (Hussong \& Chassin, 1994). In one of the first studies to examine angry mood and drinking relationships among adults, Mohr, Armeli, Tennen, and Todd (2010) utilized daily process methods and multilevel modelling, which allows for distinguishability in terms of between and within-person processes. The authors found a significant relationship between negative emotions (i.e., shame, guilt, hostility, and anger) and drinking outcomes. In regards to anger, the authors found that each unit increase of anger resulted in evening drinking by $19 \%$; in their daily diary study of alcohol, anger affect, and violence perpetration, Shorey, Stuart, Moore, and McNulty (2014) found that their female sample were more likely to physically aggress on days marked by alcohol use and angry affect. Together, these findings support the notion that daily anger is related to alcohol consumption and may result in increased drinking for veterans. 


\section{Present Study}

The purpose of the proposed thesis was to examine the temporal associations between anger and drinking behaviors in workplace veterans — an understudied population. Much research has reported on the elevated rates of anger and drinking found between service-connected individuals however, the current study aimed to contribute to findings by focusing on the within-person associations of veterans using daily diary methods. This approach not only demonstrated how anger and drinking behaviors occur within the person, but also the extent to which these relationships vary while exploring the potentially moderating role of trait anger. Such an approach provides insight into the anger-alcohol relationship as it unfolded from a daily perspective. Consequently, the present study aspired to answer the following questions:

Q1. Does daily anger predict alcohol consumption?

Q2. Does trait anger moderate the relationship between daily anger and alcohol behaviors?

Q3. If daily anger predicts alcohol consumption, do the same relationships emerge when accounting for weekend status?

Specifically, it was hypothesized that the following relationships would emerge:

Hypothesis 1a: Participants would be more likely to engage in drinking on angry days compared to non-angry days. (Within-person association)

Hypothesis $1 b$ : Participants would drink higher quantities of alcohol on days marked by higher anger relative to days marked by lower anger. (Within-person association) 
Hypothesis 2a: Participants with higher levels of trait anger would have a stronger relationship between daily anger and daily drink status compared to participants with lower levels of trait anger. (Cross-level interaction)

Hypothesis 2b: Participants with higher levels of trait anger would have a stronger relationship between daily anger and drinking quantity in comparison to individuals with lower levels of trait anger. (Cross-level interaction) 


\section{METHOD}

\section{Participants}

Five hundred and nine participants were recruited through a larger study, the Study for Employment Retention of Veterans (SERVe), a Randomized Controlled Trial (RCT) aimed at developing and enhancing supportive supervisor training in order to augment workplace culture and the well-being of veterans and their respective families. Veterans were recruited throughout 35 workplace organizations in Oregon. In order to qualify for the study, participants were required to have worked at least 20 hours per week and served in the U.S. Armed Forces since September 11, 2001. Following the SERVe study, participants were subsequently invited to participate in the Daily Family Study (DFS) sub-study, a 32-day daily diary survey assessing the health, well-being, and relationship functioning of veterans which resulted in a sample of 175 veterans after removing incomplete data. After initiating exclusion criteria, the remaining sample contained 101 participants with both SERVE and DFS data. Further exclusion criteria procedures are discussed in the data analysis section. SERVe was approved by Institutional Review Boards at Oregon Health \& Science University and Portland State University as well as the Human Research Protection Office of the U.S. Army Medical Research and Materiel Command.

Participants were comprised of veterans and reserve/guard service members who were recruited from workplace organizations within the state of Oregon. As indicated in Table 1, service-connected individuals were approximately 39 years of age $(\mathrm{SD}=9.31$ years), male (87 men, 14 women), white $(82.2 \%)$, and married $(86.1 \%)$. In regards to 
military demographics, the average participant had served almost 13 years in the military $(\mathrm{SD}=9.31)$ with over 18 months of deployment experience $(\mathrm{SD}=15.29)$. At the time of data collection, the majority of participants had been separated from the military for approximately six years $(\mathrm{SD}=3.33)$ while $18 \%$ were actively serving in the National Guard or Reserves.

\section{Baseline Measures}

Demographics. Demographic data were collected from each participant through self-report and variables included age, gender, race, education, and marital status. Military characteristics were also obtained including deployment history, duration of service, and rank.

Trait Anger. In order to measure trait anger, the Dimensions of Anger Reactions Scale (DAR; Forbes et al., 2004) Scale was used (Forbes et al., 2004). The scale was originally developed from a sample of Australian veterans with combat-related PTSD. The 7-item Likert scale ( 1 =Not at all, $8=$ Exactly so $)$ has previously demonstrated excellent reliability ( $\alpha=.91-.94$; Forbes et al., 2004) and when analyzed using data from the current sample, demonstrated excellent internal consistency $(\alpha=.91)$. The scale consists of questions such as: "I often find myself getting angry at people or situations" and "My anger prevents me from getting along with people as well as I'd like to." Scores were obtained by calculating the average score across items with higher values representing higher severity of anger. 


\section{Daily Measures}

Daily Anger. Participants completed a daily mood assessment adapted from the Positive and Negative Affect Schedule (PANAS-X, Watson \& Clark, 1994). In regards to the current study, daily anger was assessed by inquiring about the extent to which participants endorsed feeling angry at the time of measurement. Anger was measured using a 5-point Likert scale with values ranging between 1 and $5(1=$ not at all, $5=$ extremely). Fortunately, research has demonstrated that daily moods can reliably be measured with a single item (Myers \& Diener, 1995); which minimizes fatigue-related issues when daily diary methods are utilized while reducing concerns regarding whether a participant would recall their anger experiences on days in which they occur.

Daily Drinking Status and Quantity. Throughout the DFS, participants reported on the quantity of alcohol they consumed across three time-windows: between the hours of $5 \mathrm{pm}$ and 11:59pm the previous day, and between the hours of 12am - 7am and 8am $5 \mathrm{pm}$ on the current day. Given that the sample was comprised of full-time workers couple with the fact that the majority of drinking occurred during the evenings, drinks consumed in the evening were the focus of the current study. To accurately assess alcohol quantity, the daily surveys explicitly defined drinks using criteria set forth from the National Institute of Alcohol Abuse and Alcoholism (NIAAA, n.d.) with one drink being equivalent to $12 \mathrm{fl}$. oz of beer, $8-9 \mathrm{fl}$. oz of malt liquor, $5 \mathrm{fl}$. oz of wine, or $1.5 \mathrm{fl}$. oz of distilled spirits. These responses also allowed the current study to assess whether the individual drank on any particular day and the extent of their alcohol consumption; similar methods have been used in other daily diary studies of alcohol use (Armeli, 
Carney, Tennen, Affleck, \& O’Neil, 2000; Armeli, Covault, \& Tennen, 2018; Crane, Testa, Derrick, \& Leonard, 2014).

\section{Procedures}

After passing eligibility requirements, veterans consented to the study and received an electronic baseline survey. One week after completing their baseline surveys, veterans and their spouses were invited to participate in the Daily Family Study (DFS), a web-based 32-day daily diary survey assessing health, well-being, and relationship functioning for additional compensation. Baseline surveys were administered through Qualtrics and took approximately 50 minutes to complete. After completing their surveys, participants were compensated $\$ 25$. In regards to DFS procedures, surveys were sent out electronically on a daily basis throughout the duration of the study and took approximately 10 minutes to complete. Participants were instructed to complete each survey between the hours of 5PM and 11PM following their workday. To accommodate shift workers, alternative shift surveys were made available where participants were instructed to fill out their survey between the hours of 5AM and 11AM following their shifts. For their efforts, participants were compensated up to $\$ 90$ for their participation in the DFS. 


\section{DATA ANALYSES}

\section{Compliance and Missing Data}

Considering that daily diary methods are usually more taxing on the participant and involve a higher number of observations in comparison to cross-sectional surveys, special attention was devoted to study attrition and average completion rates. To

determine overall study compliance, the total number of completed surveys were divided by the total amount of survey observations possible ( $\mathrm{N}$ x 32 days). After initiating exclusion criteria (further details listed below), 101 veterans remained in the dataset, making the total number of possible observations 3,232. After obtaining descriptive statistics of the DFS data, it was found that on average, participants completed 24 days resulting in a total of 2,302 responses-yielding a compliance rate of $71 \%(2,302 / 3,232)$. While many concerns can be posed over the prospect of missing data, multilevel approaches are robust to missing data and various software packages offer a variety of estimation approaches thus, concerns of missing data were mitigated.

The fact that some individuals had higher rates of reporting compared to others, created unequal group sizes or unbalanced design but fortunately, multilevel regression approaches are known for managing unbalanced data well using maximum likelihood (FIML) estimation, which proffers better estimations than traditional missing data solutions and allows for comparing nested models (Raudenbush \& Bryk, 2002). Another added benefit of analyzing the data with a multilevel regression approach was the fact that participants with greater response rates had higher weighted proportions and thus influenced the average relationships observed to a greater degree compared to those 
whose responses were sparse. The original DFS dataset consisted of 175 veterans. To ensure that the non-drinking behaviors of alcohol abstainers did not have an undue effect on the temporal associations between daily anger and evening drinking, individuals who failed to report at least one drink throughout the DFS were removed from the dataset and resulted in the removal of 38 participants—similar procedures have been used in previous daily diary studies (e.g., McCabe, 2016). Given the fact that each day, participants reported their daily anger and their drinking behaviors from the evening prior, there was a need to ensure that analyses could draw from successive daily reports. Therefore, in an effort to address this issue and better manage missing data, participants who did not have at least two days of consecutive data were removed from the dataset and resulted in the removal of 9 participants. Finally, the primary focus was on individuals who worked dayshifts as they made up the majority of the sample, therefore, individuals who worked the nightshift were excluded from the analyses resulting in the removal of 27 participants. It is important to note that some research has found that shift workers experience occasional or chronic sleep problems (Åkerstedt, 2003) and individuals who do not sleep well may engage in drinking for its sedative effects (Soldatos et al., 2005). Moreover, it has also been reported that shift workers have disparate drinking behaviors (Dorrian \& Skinner, 2012) when compared to dayworkers which strengthens the argument for removing shift workers from the current study.

Power

When considering minimum sample size and power within a multilevel analysis framework, the number of groups is more influential than the number of cases per group 
(Scherbaum \& Ferreter, 2009). Recent findings have suggested that having 50 or more groups with 5-10 cases per group will generally assist in avoiding issues with convergence and estimation biases (McNeish \& Stapleton, 2014) although these criteria vary based on the study design. The DFS was comprised of 175 veterans who reported on daily events over the course of 32 days. After exclusion criteria was implemented, analyses revealed that the number of clusters (i.e., veterans) varied between 94 and 98 therefore, the prospect of possessing adequate power is likely.

\section{Variable Management}

Before the data were analyzed, several outcome variables needed to be created. Evening Drink Status was computed by observing whether an individual consumed alcohol during the evening - if the participant reported at least one drink, their evening drink status was coded as 1 for that day (otherwise, that day's observation was coded as 0). Considering that evening drinking data was recorded on the subsequent day of each daily survey, individual anger was lagged by participant ID by one day such that afternoon anger predicted that evening's drinking as reported on the following day. This approach allowed for the prediction of evening alcohol consumption using daily anger while controlling for weekend status and consequently, bolstered the temporal association. To aid in the interpretability of results, predictors were rescaled through centering. Specifically, the within-person variables were person-centered while betweenperson variables were grand-mean centered as suggested by Raudenbush and Bryk (2002). Level-one predictors consisted of daily anger and weekend/weekday status $($ Sunday - Thursday $=0$; Friday $\&$ Saturday $=1)$ as previous research has reported trends 
on day-of-week and alcohol consumption (Mohr et al., 2001; Armeli et al., 2000). In terms of between-person model specifications, trait anger was utilized as a level-two variable.

\section{Preliminary Analyses}

Before testing the stated hypotheses, descriptive statistics and Pearson correlations were obtained for all variables of interest in a preliminary attempt to inspect the data. In order to assess daily responses, correlation matrices were obtained by aggregating the daily values across the 32-day period. Given that the dependent variables were comprised of nonlinear data (i.e., binary, count), distributions of the variables were obtained through the use of scatterplots. Outliers were detected using Mahalanobis distance, Cook's distance, and boxplots. Outliers are generally identified by Cook's distance (values greater than 1; Cook \& Weisberg, 1982) as well as Mahalanobis distance (values greater than 11; Barnett \& Lewis, 1978). While these outlier tests were developed with ordinary multiple regression models in mind, Tabachnick and Fidell (1996) suggest that they can be used to determine outliers within multilevel models as well. Although many conventional practices result in the removal of outliers when conducting betweenperson analyses in order to prevent biasing results, these considerations are of less concern when conducting within-person analyses and thus, outliers were retained. 


\section{RESULTS}

\section{Descriptive Statistics}

Descriptive analyses (Table 2) also revealed that in terms of trait anger, participants on average, had an average score of close to $3(\mathrm{M}=2.75, \mathrm{SD}=1.48)$, suggesting that most participants had lower levels of trait anger. In terms of daily measures, participants on average, consumed 1 drink in the evening $\left(\mathrm{M}_{\text {drink }}=1.24, \mathrm{SD}=\right.$ 1.73). The average level of anger reported while completing their daily surveys was 1.29 $(\mathrm{SD}=.51)$ indicating that participants were experiencing relatively low levels of anger while completing daily surveys. In regard to evening drink status, preliminary analyses indicated that across the 32-days, $50.7 \%$ of the daily reports indicated evening drinkingthe majority of which, occurred on non-weekend evenings (i.e., Sunday-Thursday, $71.3 \%)$

\section{Multilevel Analyses}

In order to test the proposed hypotheses, data were analyzed using multilevel regression and carried out in Mplus version 8 (Muthén \& Muthén, 1998-2017) to investigate the between- and within-person effects of daily anger on alcohol consumption. The analytical approach was appropriate given the nested structure of daily diary measures occurring within the individual. In order to account for "weekend drinking effects," weekend vs. weekday status was included in the within-model as a time-varying covariate. It is important to note that the dependent variables used in this study were not continuous. Evening Drink Status follows a binary structure $(0=$ no, 1=yes); therefore, multilevel logistic models predicting this particular outcome were used 
to test the models. Considering that number of evening drinks falls under the criteria of count data, assumptions of data normality were violated (Gardner, Mulvey, \& Shaw, 1995); therefore, negative binomial models were used to test all analyses involving count data as a dependent variable. Specifically, Zero-Inflated Negative Binomial (ZINB) regression models were utilized and are often appropriate when using multilevel count data with a preponderance of zero values. While Zero-Inflated Poisson (ZIP) models could also have been used, studies have found that ZINB models are more robust, handle overdispersed data more efficiently, and are superior in modeling zero counts in comparison to ZIP models (UCLA, n.d.; Zeileis, Kleiber, \& Jackman, 2008).

In sum, two-level models were tested in Mplus using MLR estimation to account for both non-normal and missing data which produces robust standard errors through the use of sandwich estimation (Muthén \& Muthén, 1998-2017). As mentioned previously, it was suspected that the outcome variables would most likely violate assumptions of independent observations considering the clustered nature of the analyses. Preliminary models in Mplus produced Intraclass Correlation Coefficients (ICCs) for the outcome variables of interests. ICCs were .40 for evening drinks and .28 for evening drink status providing further evidence that multilevel regression was indeed appropriate.

Hypothesis 1. Hypothesis 1a posited that participants would be more likely to drink on angry days compared to non-angry days. In order to test this hypothesis, withinin person associations between daily anger and alcohol use were determined by regressing evening drink status on daily anger. As seen in Table 3 , results indicated that 
daily anger did not significantly predict evening drink status, controlling for weekend/weekday status, $b=.08, S E=.09, p=\mathrm{ns}$.

Hypothesis $1 \mathrm{~b}$ stated that individuals would consume higher quantities of alcohol on days marked by higher anger compared to days with lower anger. This within-person relationship was determined by regressing alcohol quantity on to daily anger. This hypothesis was partially supported in that the relationship emerged albeit in the opposite hypothesized direction. Results indicated that daily anger negatively predicted number of evening drinks, $b=-.10, S E=.05, p<.05$.

Hypothesis 2. Hypothesis 2a posited that individuals with higher levels of trait anger would have a stronger positive relationship between daily anger and evening drink status in comparison to individuals with low levels of trait anger. These associations were tested by observing cross-level interactions between trait anger (level 2) and daily anger (level 1) and whether their interaction influences the evening drink status outcome variable. The created interaction term of daily anger and trait anger had a marginally significant association with evening drink status, $b=.13, S E=.07, p=.053$, providing some evidence that trait anger moderates the relationship between daily anger and evening drink status. Despite the nonsignificant finding, simple slopes were obtained to further probe the relationship and determine whether patterns were consistent with the hypothesis. Simple slopes were tested by restraining levels of trait anger to include values at the mean as well as 1 standard deviation above and below the mean. Subsequent analyses indicated that low trait anger (-1 SD) had a marginally significant slope $b=$ - 
$.30, S E=.15, p=.051$, whereas slopes for mean and high trait anger were nonsignificant, $b=-.10, S E=.11, p=\mathrm{ns} ; b=.10, S E=.15, p=\mathrm{ns}$, respectively.

H2b: Finally, Hypothesis $2 b$ stated that participants with higher levels of trait anger will have a stronger positive relationship between daily anger and drinking quantity compared to individuals with lower levels of trait anger. This hypothesis was also tested by observing the cross-level interaction between trait anger (level 2) and daily anger (level 1) and their influence on alcohol quantity. The interaction term was significantly related to number of evening drinks, $b=.11, S E=.03, p<.01$, suggesting that the daily anger-drinking quantity relationship varied as a function of trait anger. Simple slopes were tested using values of trait anger at the mean, and one standard deviation above and below the mean, revealing that participants at low and mean levels of trait anger had significant negative slopes $(b=-.39, S E=.16, p<.05 ; b=-.21, S E=.05, p<.001$, respectively), whereas those participants with high trait anger did not show a significant daily anger-drinking quantity relationship, $b=-.05, S E=.05, p=\mathrm{ns}$. 


\section{DISCUSSION}

The current study aimed to examine the daily associations found between anger and drinking behaviors among separated service members and active reservists using a daily diary design. The proposed hypotheses of the current study were partially supported by the findings. The first hypothesis (H1a) proposed that daily anger would predict evening drink status. However, hypothesis 1a was not supported. The second hypothesis (H1b) posited that daily anger would be positively associated with the number of evening drinks consumed while controlling for weekend status. Findings indicated that these relationships did emerge, although in the opposite direction than hypothesized. Specifically, daily anger was negatively related to the number evening drinks consumed by participants indicating that on angry days, participants consumed less alcohol. The third hypothesis $(\mathrm{H} 2 \mathrm{a})$ proposed that trait anger would moderate the relationship between daily anger and evening drink status. Although trait anger was not significantly related to whether or not participants drank on a given evening there was some evidence, in the form of a marginally-significant interaction, that the relationship between daily anger and evening drinks status may vary across level of trait anger. The final hypothesis $(\mathrm{H} 2 \mathrm{~b})$ posited that the relationship between daily anger and number of evening drinks would be moderated by trait anger, which was affirmed. However, the form of the interaction was counter to prediction, in which participants at mean- and low-levels of trait anger consumed less alcohol following afternoons of higher daily anger. Surprisingly, participants with higher levels of trait anger were not more likely to drinking more on evenings following increases in daily anger. 
The present study was one of the first to examine the within-person drinking associations between anger and alcohol among service-connected individuals. Overall, the findings of the study shed light on the intrapersonal drinking behaviors of veterans with results suggesting that daily anger is negatively related to the number of evening drinks consumed within a sample of veterans - especially among those with lower levels of trait anger. Surprisingly, higher levels of trait anger had no bearing on the association between daily anger and evening drinking quantity however, it should be noted that average levels of trait anger found within the sample were relatively low and these patterns may not emerge in veteran samples with higher levels of anger (e.g., patients with PTSD). The study also elucidates drinking behaviors in a non-clinical veteran sample, which is not well-represented in the literature with results suggesting that many veterans are not drinking heavily on days in which they are angry. Finally, the current study demonstrates how the interplay between anger and drinking may vary across individuals with varying levels of trait anger. Specifically, veterans with average and low levels of trait anger may have lower motivations to drink when angry. It is also possible that participants may have been saving their drinks for the weekend and may therefore have different anger-drinking patterns between weekday anger and weekend drinking in comparison to the current tested relationship. Alternatively, it may be that these veterans were efficiently regulating their emotions when provoked or could be engaging in healthier coping mechanisms to attenuate the effects of anger. For example, some evidence has emerged suggesting that cognitive behavioral interventions such as relaxation, coping, and communication skills have been efficacious in attenuating anger 
and aggression-related problems within different populations (Blake \& Hamrin, 2007). Some interventions utilizing expressive writing have shown promising results in reducing anger, attenuating cortisol levels, and enhancing emotion regulation skills among PTSD patients and may aid in regulating psychological distress and trauma related thoughts (Smyth, Hockemeyer, \& Tulloch, 2008). It should be mentioned however, that further research from longitudinal and daily perspectives is needed to clarify the relationship between anger and alcohol use among veterans. For example, combat veterans with recent deployment experiences could theoretically have disparate anger-drinking patterns in comparison to the current sample as deployment experiences among veterans is often associated with increased risk for anger outbursts and alcohol dependence (Tanielian, Batka, \& Meredith, 2017).

It is also unclear whether some of these salubrious behaviors are influenced by past or present military training. For example, Master Resilience Training (MRT) has been provided to soldiers since 2009 and is currently a mandatory training requirement in the U.S. Army. In their investigation of wellbeing and stressors among National Guardsman, Griffith and West (2013) reported that 92\% of participants who had completed MRT believed that their resilience training was helpful in coping with stress and $97 \%$ indicated that they used their resilience skills within their military and civilian roles. In essence, the degree to which veterans are engaging in these health-promoting behaviors is unclear, and research could benefit from future investigation in order to clarify the presence of these effects. Such approaches may elucidate the resilience of veterans long after military service. 
The vast majority of participants were married and had been in long-term relationships for an average of 11 years. It is possible that relationship factors could have served as a stress buffer and provided the veteran with social support and alternative forms of stress-relief such as dyadic coping (Meuwly et al., 2012) which in turn, reduced their reliance on alcohol when experiencing anger. Examining how relationship factors influence anger may therefore, provide further insight into the daily behaviors of veterans in the workplace.

Finally, it may be prudent for future studies to replicate the proposed study while differentiating between the various forms of anger (i.e., Anger-In, Anger-Out, State, and Trait). For example, some research has shed light on the positive effects of expressed anger (i.e., anger-out) in the workplace (Geddes \& Callister, 2007), which presumably, may influence an individual's daily drinking behavior. Subsequent studies should also examine the directionality of the proposed findings to clarify the temporal relationship between anger and drinking behaviors while documenting whether the same relationships emerge by gender as well as in young, single, and recently-separated veterans.

\section{Study Limitations}

There are several limitations of the proposed study. All of the measures were obtained through self-report and thus, run the risk of misreporting. Considering that alcohol quantities tend to be underreported (Stockwell et al., 2004), it is possible that the current findings are conservative estimates. However, Perrine, Mundt, Searless, and Lester (1995) found good concordance between self-reports, collateral reports, and blood alcohol content (BAC) levels within a daily study — suggesting that retrospective self- 
reported drinking within a 24-hour period is highly correlated with objective measures of alcohol use. This concern is also attenuated when considering the within-person nature of the study where responses were nested within the individual thus, each person served as their own control while emphasis was placed on the deviations of their normative drinking behaviors.

In addition, the current study utilized a daily diary design over the course of 32days and responses were reported throughout the day as the survey used an intervalcontingent recording method. The fact that momentary mood was assessed could be perceived as a limitation and alternatively, participants could have reported their mood throughout the day. Although, this approach may have led to participant fatigue over the 32-day period and some evidence has suggested that an individual's emotion recall is heavily influenced by their current mood (Natale \& Hantis, 1982), which may not result in significant differences between the two methods. It is also worth highlighting a particular strength of the study in that mood and drinking data were collected on separate surveys which reduces concerns of common method bias while providing conservative estimates of their relationship.

Some of the moderation analyses conducted within the current study revealed marginally significant results. While previous sections provided rationale for meeting minimum sample sizes requirements in order to test main effects of nested models, some authors recommend a sample size of 100-200 groups to test cross-level interactions (Hox, 2010). Therefore, the cross-level interactions within the present study may have been underpowered. A final limitation of the study centered around the demographics of 
veterans surveyed. As indicated earlier, most of the sampled veterans were approximately 39 years old, married, and had been separated from the military for 6 years on average. All of our participants were gainfully employed and although the analyses were comprised of drinkers as ensured through the selection criteria, the subset of employed veterans in our sample may have intrapersonal drinking behaviors that do not generalize to other veteran groups such as young, single, or newly-separated service members.

\section{Conclusion}

The field of veteran health research has made remarkable strides in separately documenting the elevated rates of anger and drinking found among service-connected individuals. Although some civilian studies have explicitly examined the relationship between the two constructs, few have explored the phenomenon using a within-person approach from a multilevel framework. The present study is the first to examine the intrapersonal relationship between anger and drinking behaviors among veterans and provides multiple implications. First, this study observed relatively low rates of daily anger, trait anger, and alcohol consumption found among veterans. This is contrary to the belief that problems associated with anger and alcohol abuse follow the veteran many years after military separation. Second, these findings suggest that workplace veterans may drink less alcohol when angry, especially among individuals with low and average levels of trait anger. This finding may better inform prospective employers by demonstrating the resilient behaviors of employed veterans with notable deployment experiences while assisting practitioners by identifying lower-risk drinkers through the 
use of their respective trait anger levels. Finally, the current study may encourage the use of anger reduction techniques in order to curb alcohol use in veteran samples. Given that anger and alcohol misuse are highly prevalent in veteran samples, these findings present a unique opportunity to address both issues in concert. In sum, service-connected individuals have endured multiple challenges, from the stressors associated with military life and combat, to the hardships associated with civilian reintegration. It is evident that one common theme emerges: their future wellbeing is not necessarily tied to their past adversities. 
Table 1

Sociodemographic and Military Characteristics

\begin{tabular}{lr} 
Variable & $\mathrm{M}(\mathrm{SD}) / \%$ \\
\hline Age & $38.97(9.31)$ \\
Male & $86.1 \%$ \\
White & $82.2 \%$ \\
College graduate & $74.2 \%$ \\
Married & $86.1 \%$ \\
Active in the military & $18.8 \%$ \\
Years in the military & $12.63(8.10)$ \\
Enlisted & $76.2 \%$ \\
Years since separated from military & $5.80(3.33)$ \\
Deployment Experience (Total Months) & $18.55(15.29)$ \\
\hline
\end{tabular}

Note: $\mathrm{N}=101$ 
Table 2

Descriptives and Inter-Correlations Among Study Variables

\begin{tabular}{lccccccc}
\hline Variable & Mean/\% & SD & 1 & 2 & 3 & 4 & 5 \\
\hline 1. Anger & 1.29 & 0.51 & - & & & \\
2. Wkdstat & $28.7 \%$ & N/A & .00 & - & & \\
3. TA & 2.75 & 1.48 & $\mathbf{. 1 8}$ & .00 & - & & \\
4. Edkstat & $50.7 \%$ & N/A & $\mathbf{. 0 8}$ & .04 & $\mathbf{- . 0 4}$ & - & \\
5. Drinks & 1.24 & 1.73 & $\mathbf{. 1 1}$ & $\mathbf{. 0 4}$ & $\mathbf{- . 0 6}$ & $\mathbf{. 7 0}$ & - \\
\hline
\end{tabular}

Note: Anger $=$ Daily Anger. Wkdstat $=$ Weekend Status. TA $=$ Trait Anger.

Edkstat $=$ Evening Drink Status. Drinks $=$ Number of Evening Drinks. 
Table 3

Multilevel Regression Results for Study Variables

\begin{tabular}{ccccc}
\hline \multicolumn{2}{c}{$\begin{array}{c}\text { Model 1 } \\
\text { Edkstat }\end{array}$} & \multicolumn{2}{c}{$\begin{array}{c}\text { Model 2 } \\
\text { Drinks }\end{array}$} \\
\hline$b$ & $S E$ & $b$ & $S E$
\end{tabular}

Within-Person Effects

$\begin{array}{lcccc}\text { Anger } & .08 & .09 & -.10^{*} & .05 \\ \text { Wkdstat } & .33^{*} & .14 & .07^{*} & .04\end{array}$

Threshold

$-.09 \quad .19$

Variance

2.77

.55

.92

.14

\begin{tabular}{lrrrr}
\hline & \multicolumn{2}{c}{$\begin{array}{c}\text { Model 3 } \\
\text { Edkstat }\end{array}$} & \multicolumn{2}{c}{$\begin{array}{c}\text { Model 4 } \\
\text { Drinks }\end{array}$} \\
\hline$b$ & $S E$ & SE & $S E$
\end{tabular}

Within-Person Effects

\begin{tabular}{lcccc} 
Anger & -.09 & .11 & $-.21^{* * *}$ & .05 \\
Wkdstat & $.33^{*}$ & .16 & .10 & .08 \\
Anger X TA & .13 & .07 & $.11^{* *}$ & .03 \\
Between-Person Effect & -.06 & .12 & -.03 & .15 \\
TA & -.12 & .19 & - & - \\
Threshold & - & - & .02 & .14 \\
Intercept & 2.43 & .64 & .81 & .16 \\
Residual Variance & & & & \\
\hline
\end{tabular}

Note $:$ Anger $=$ Daily Anger. Wkdstat $=$ Weekend Status. TA $=$ Trait Anger .

Edkstat $=$ Evening Drink Status. Drinks $=$ Number of Evening Drinks.

$* \mathrm{p}<.05 * * \mathrm{p}<.01 * * * \mathrm{p}<.001$ 
Figure 1. Hypothesized Model

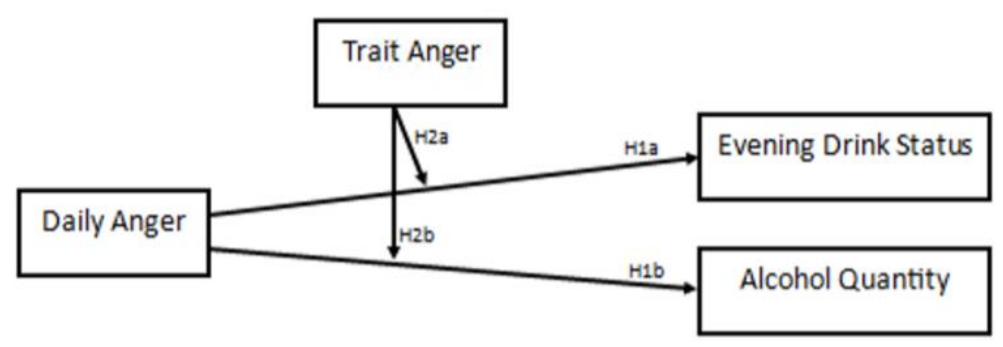


Figure 2. DFS Daily Login Procedures

\begin{tabular}{|c|c|c|c|c|}
\hline $\begin{array}{c}\text { Yesterday 5-12PM } \\
\# \\
\text { Of Drinks }\end{array}$ & 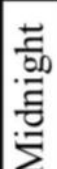 & $\begin{array}{c}\text { Today 12am-7am } \\
\# \\
\text { Of Drinks }\end{array}$ & $\begin{array}{c}\text { Today } 8 \mathrm{am}-5 \mathrm{pm} \\
\# \\
\text { Of Drinks }\end{array}$ & Today $5 \mathrm{pm}-11 \mathrm{pm}$ \\
\hline
\end{tabular}




\section{REFERENCES}

Adler, A. B., Brossart, D. F., \& Toblin, R. L. (2017). Can anger be helpful?: Soldier perceptions of the utility of anger. Journal of Nervous and Mental Disease, 205(9), 692-698. https://doi.org/10.1097/NMD.0000000000000712

Åkerstedt, T. (2003). Shift work and disturbed sleep/wakefulness. Occupational medicine, 53(2), 89-94.

Anderson, C. A., \& Bushman, B. J. (2002). Human aggression. Annual review of psychology, 53, 27-51.

Andersson, L. M., \& Pearson, C. M. (1999). Tit for tat? The spiraling effect of incivility in the workplace. Academy of Management Review, 24, 452-471.

Armeli, S., Carney, M. A., Tennen, H., Affleck, G., \& O'Neil, T. P. (2000). Stress and alcohol use: A daily process examination of the stressor-vulnerability model. Journal of personality and social psychology, 78(5), 979.

Armeli, S., Covault, J., \& Tennen, H. (2018). Long-term changes in the effects of episode-specific drinking to cope motivation on daily well-being. Psychology of Addictive Behaviors, 32(7), 715.

Averill, J. A. (1982). Anger and Aggression: An Essay on Emotion. New York, NY: Springer-Verlag. https://doi.org/10.1007/978-1-4612-5743-1.

Bandura, A. (1978). Social learning theory of aggression. Journal of communication, 28(3), 12-29.

Barnett, V., \& Lewis, T. (1978). Outliers in statistical data. NewYork: Wiley. 
Baumeister, R. F., Vohs, K. D., \& Tice, D. M. (2007). The strength model of self-control. Current directions in psychological science, 16(6), 351-355.

Berkowitz, L. (1989). Frustration-Aggression Hypothesis: Examination and Reformulation. Psychological Bulletin, 106(1), 59-73. https://doi.org/00332909/89.

Berkowitz, L. (2012). A different view of anger: The cognitive-neoassociation conception of the relation of anger to aggression. Aggressive behavior, 38(4), 322-333.

Blake, C. S., \& Hamrin, V. (2007). Current approaches to the assessment and management of anger and aggression in youth: A review. Journal of Child and Adolescent Psychiatric Nursing, 20(4), 209-221.

Bliese, P. D., Wright, K. M., Adler, A. B., Cabrera, O., Castro, C. A., \& Hoge, C. W. (2008). Validating the primary care posttraumatic stress disorder screen and the posttraumatic stress disorder checklist with soldiers returning from combat. Journal of consulting and clinical psychology, 76(2), 272.

Bliese, P. D., Wright, K. M., Adler, A. B., Thomas, J. L., \& Hoge, C. W. (2007). Timing of postcombat mental health assessments. Psychological Services, 4(3), 141-148.

Booth, J., \& Mann, S. (2005). The experience of workplace anger. Leadership \& Organization Development Journal, 26(4), 250-262. https://doi.org/10.1108/01437730510600634. 
Bray, R., Marsden, M. \& Peterson, M. (1991). Standardized comparisons of the use of alcohol, drugs, and cigarettes among military personnel and civilians. American Journal of Public Health, 81, 865 - 869.

Bray, R. M., Pemberton, M. R., Lane, M. E., Hourani, L. L., Mattiko, M. J., \& Babeu, L. a. (2010). Substance Use and Mental Health Trends Among U.S. Military Active Duty Personnel: Key Findings From the 2008 DoD Health Behavior Survey. Military Medicine, 175(6), 390-399. https://doi.org/10.7205/MILMED-D-0900132

Browne, T., Iversen, A., Hull, L., Workman, L., Barker, C., Horn, O., ... \& Hotopf, M. (2008). How do experiences in Iraq affect alcohol use among male UK armed forces personnel? Occupational and environmental medicine, 65(9), 628-633.

Castillo, D. T., Baca, J. C. D., Conforti, K., Qualls, C., \& Fallon, S. K. (2002). Anger in PTSD: General psychiatric and gender differences on the BDHI. Journal of Loss \&Trauma, 7(2), 119-128.

Chemtob, C. M., Novaco, R. W., Hamada, R. S., Gross, D. M., \& Smith, G. (1997). Anger Regulation Deficits in Combat-Related Posttraumatic Stress Disorder. Journal of Traumatic Stress, 10(1), 17-36.

Christiansen, P., Cole, J. C., \& Field, M. (2012). Ego depletion increases ad-lib alcohol consumption: Investigating cognitive mediators and moderators. Experimental and clinical psychopharmacology, 20(2), 118. 
Conger, J. J. (1956). Alcoholism: Theory, problem and challenge. II. Reinforcement theory and the dynamics of alcoholism. Quarterly Journal of Studies on Alcohol, $17,296-305$.

Crane, C. A., Testa, M., Derrick, J. L., \& Leonard, K. E. (2014). Daily associations among self-control, heavy episodic drinking, and relationship functioning: An examination of actor and partner effects. Aggressive behavior, 40(5), 440-450.

Cook, R. D., \& Weisberg, S. (1982). Residuals and influence in regression. New York: Chapman \& Hall.

Cooper, M. L. (1994). Motivations for alcohol use among adolescents: Development and validation of a four-factor model. Psychological Assessment, 6, 117-128. doi:10.1037/1040-3590.6.2.117

Cooper, M. L., Frone, M. R., Russell, M., \& Mudar, P. (1995). Drinking to regulate positive and negative emotions: A motivational model of alcohol use. Journal of Personality and Social Psychology, 69, 990-1005. doi:10.1037/00223514.69.5.990

Cox, D. E., \& Harrison, D. W. (2008). Models of anger: Contributions from psychophysiology, neuropsychology and the cognitive behavioral perspective. Brain Structure and Function, 212(5), 371-385. https://doi.org/10.1007/s00429007-0168-7

Dafoe, A., \& Caughey, D. (2016). Honor and War. World Politics, 68(2), 341-381. https://doi.org/10.1017/S0043887115000416 
Deffenbacher, J. L., Oetting, E. R., \& DiGiuseppe, R. A. (2002). Principles of empirically supported interventions applied to anger management. The Counseling Psychologist, 30(2), 262-280.

Defense Manpower Data Center. (2010). June 2009 status of forces survey of reserve component members: Family life briefing (unpublished report). (DMDC, Note N. 2010-013).

Dollard, J., Miller, N. E., Doob, L. W., Mowrer, O. H., \& Sears, R. R. (1939). Frustration and aggression.

Dorrian, J., \& Skinner, N. (2012). Alcohol consumption patterns of shiftworkers compared with dayworkers. Chronobiology international, 29(5), 610-618.

Elder, G. H., Hill, C., Wang, L., Hill, C., Adkins, D. E., \& Brown, T. H. (2010). Pathways to the All-Volunteer Military, Social Science Quarterly, 91(2), 455-475.

Feeny, N. C., Zoellner, L. A., \& Foa, E. B. (2000). Anger, dissociation, and posttraumatic stress disorder among female assault victims. Journal of Traumatic Stress, 13(1), 89-100.

Forbes, D., Hawthorne, G., Elliott, P., McHugh, T., Biddle, D., Creamer, M., \& Novaco, R. W. (2004). A concise measure of anger in combat-related posttraumatic stress disorder. Journal of Traumatic Stress: Official Publication of The International Society for Traumatic Stress Studies, 17(3), 249-256. 
Forbes, D., Parslow, R., Creamer, M., Allen, N., McHugh, T., \& Hopwood, M. (2008). Mechanisms of anger and treatment outcome in combat veterans with posttraumatic stress disorder. Journal of Traumatic Stress, 21(2), 142-149. https://doi.org/10.1002/jts.20315

Gardner, W., Mulvey, E. P., \& Shaw, E. C. (1995). Regression analyses of counts and rates: Poisson, overdispersed Poisson, and negative binomial models. Psychological bulletin, 118(3), 392.

Geddes, D., \& Callister, R. R. (2007). Crossing the line (s): A dual threshold model of anger in organizations. Academy of Management Review, 32(3), 721-746.

Gianakos, I. (2002). Issues of anger in the workplace: Do gender and gender role matter? Career Development Quarterly, 51, 155-171.

Glomb, T. M. (2002). Workplace anger and aggression: informing conceptual models with data from specific encounters. Journal of occupational health psychology, 7(1), 20-36.

Glomb, T. M., \& Hulin, C. L. (1997). Anger and gender effects in observed supervisorsubordinate dyadic interactions. Organizational behavior and human decision processes, 72(3), 281-307.

Griffith, J. \& West, C. (2013). Master resilience training and its relationship to individual well -being and stress buffering among army national guard soldiers. Journal of Behavioral Health Services \& Research, 40(2), 140-155. 
Hawthorne, G., Mouthaan, J., Forbes, D., \& Novaco, R. W. (2006). Response categories and anger measurement : do fewer categories result in poorer measurement? Social Psychiatry and Psychiatric Epidemiology, 41, 164-172. https://doi.org/10.1007/s00127-005-0986-y

Heesink, L., Rademaker, A., Vermetten, E., Geuze, E., \& Kleber, R. (2015). Longitudinal measures of hostility in deployed military personnel. Psychiatry research, 229(12), 479-484.

Hoge, C. W., Castro, C. A., Messer, S. C., McGurk, D., Cotting, D. I., \& Koffman, R. L. (2004). Combat Duty in Iraq and Afghanistan, Mental Health Problems, and Barriers to Care. The New England Journal of Medicine, 351(1), 13-22. https://doi.org/10.1056/NEJMoa040603

Hox, J. (2010). Multilevel analysis: Techniques and applications, second edition. New York: Routledge.

Hussong, A. M., \& Chassin, L. (1994). The stress-negative affect model of adolescent alcohol use: disaggregating negative affect. Journal of studies on alcohol, 55(6), 707-718.

Jackson, J. J., Thoemmes, F., Jonkmann, K., Lüdtke, O., \& Trautwein, U. (2012). Military training and personality trait development: Does the military make the man, or does the man make the military? Psychological Science, 23(3), 270-277. https://doi.org/10.1177/0956797611423545 
Jakupcak, M., Conybeare, D., Phelps, L., Hunt, S., Holmes, H. A., Felker, B., ... McFall, M. E. (2007). Anger, hostility, and aggression among Iraq and Afghanistan war veterans reporting PTSD and subthreshold PTSD. Journal of Traumatic Stress, 20(6), 945-954. https://doi.org/10.1002/jts.20258

Kessler, R. C., Heering, S. G., Stein, M. B., Colpe, L. J., Fullerton, C. S., Hwang, I., Naifeh, J. A., Nock, M. K., Petukhova, M., Sampson, N. A., Schoenbaum, M., Zaslavsky, A. M., \& Ursano, R. J., Army STARRS Collaborators (2014) Thirtyday prevalence of DSM-IV mental disorders among non-deployed soldiers in the U.S. Army: Results from the Army Study to Assess Risk and Resilience in Servicemembers (Army STARRS). JAMA Psychiatry, 71, 487-489.

Khantzian, E. J. (2003). The self-medication hypothesis revisited: The dually diagnosed patient. Primary Psychiatry, 10(9), 47-54.

Langdon, K. J., Fox, A. B., King, L. A., King, D. W., Eisen, S., \& Vogt, D. (2016). Examination of the dynamic interplay between posttraumatic stress symptoms and alcohol misuse among combat-exposed Operation Enduring Freedom (OEF)/Operation Iraqi Freedom (OIF) Veterans. Journal of affective disorders, 196, 234-242.

Lindebaum, D., Jordan, P. J., \& Morris, L. (2016). Symmetrical and asymmetrical outcomes of leader anger expression: A qualitative study of army personnel. Human Relations, 69(2), 277-300. https://doi.org/10.1177/0018726715593350. 
Linkh, D. J., \& Sonnek, S. M. (2003). An application of cognitive-behavioral anger management training in a military/occupational setting: Efficacy and demographic factors. Military medicine, 168(6), 475-478.

Meier, B. P., Robinson, M. D., \& Wilkowski, B. M. (2006). Turning the Other Cheek: Agreeableness and the Regulation of Aggression-Related Primes. Psychological Science, 17(2), 136-142. http://dx.doi.org/10.1111/j.1467-9280.2006.01676.x

Meuwly, N., Bodenmann, G., Germann, J., Bradbury, T. N., Ditzen, B., \& Heinrichs, M. (2012). Dyadic coping, insecure attachment, and cortisol stress recovery following experimentally induced stress. Journal of Family Psychology, 26(6), 937.

Miller, S. M., Pedersen, E. R., \& Marshall, G. N. (2017). Combat experience and problem drinking in veterans: Exploring the roles of PTSD, coping motives, and perceived stigma. Addictive Behaviors, 66, 90-95. https://doi.org/10.1016/j.addbeh.2016.11.011

McCabe, Cameron Trim, "Vulnerability and Protective Factors of Stress-Related Drinking: An Exploration of Individual and Day-Level Predictors of Alcohol Involvement" (2016). Dissertations and Theses. Paper 3287.

McCabe, C. T., Mohr, C. D., Hammer, L. B., \& Carlson, K. F. (2019). PTSD symptomology and motivated alcohol use among military service members: testing a conditional indirect effect model of social support. Substance use \& misuse, 54(2), 257-270. 
McNeish, D., \& Stapleton, L. (2014). The Effect of Small Sample Size on Two-Level Model Estimates: A Review and Illustration. Educational Psychological Review, 1-20. http://dx.doi.org/10.1007/s10648-014-9287-X

Mohr, C.D., Armeli, S., Tennen, H., Carney, M., Affleck, G. \& Hromi, A. (2001). Daily interpersonal experiences, context and alcohol consumption: Crying in your beer and toasting good times. Journal of Personality and Social Psychology, 80 (3), 489-500.

Mohr, C., Armeli, S., Tennen, H., \& Todd, M. (2010). The complexities of modeling mood-drinking relationships: Lessons learned from daily process research. In J. D. Kassel (Ed.), Substance abuse and emotion (p. 189-216). American Psychological Association. https://doi.org/10.1037/12067-008

Mohr, C. D., McCabe, C. T., Haverly, S. N., Hammer, L. B., \& Carlson, K. F. (2018). Drinking motives and alcohol use: The SERVe Study of US current and former service members. Journal of studies on alcohol and drugs, 79(1), 79-87.

Muraven, M., Collins, R. L., \& Neinhaus, K. (2002). Self-control and alcohol restraint: an initial application of the self-control strength model. Psychology of Addictive Behaviors, 16(2), 113.

Muthén, L.K. and Muthén, B.O. (1998-2017). Mplus User’s Guide. Eighth Edition. Los Angeles, CA: Muthén \& Muthén

Myers, D. G., \& Diener, E. (1995). Who is happy? Psychological science, 6(1), 10-19. 
Natale, M., \& Hantas, M. (1982). Effect of temporary mood states on selective memory about the self. Journal of Personality and Social Psychology, 42(5), 927.

National Institute on Alcohol Abuse and Alcoholism. (NIAAA, n.d.). Retrieved from https://www.niaaa.nih.gov/what-standard-drink

Nisbett, R.E., \& Cohen, D. (1996). Culture of honor: The psychology of violence in the South. Boulder, CO: Westview Press.

O’Neill, O. A., Vandenberg, R. J., DeJoy, D. M., \& Wilson, M. G. (2009). Exploring Relationships Among Anger, Perceived Organizational Support, and Workplace Outcomes. Journal of Occupational Health Psychology, 14(3), 318-333. https://doi.org/10.1037/a0015852.

Perrine, M. W., Mundt, J. C., Searles, J. S., \& Lester, L. S. (1995). Validation of daily self-reported alcohol consumption using interactive voice response (IVR) technology. Journal of Studies on Alcohol, 56(5), 487-490.

RAND Corporation. (2019). Improving the Quality of Mental Health Care for Veterans: Lessons from RAND Research. https://www.rand.org/pubs/research_briefs/RB10087.html

Reyes, V. A., \& Hicklin, T. A. (2005). Anger in the Combat Zone. Military Medicine. https://doi.org/10.7205/milmed.170.6.483.

Raudenbush, S. W., \& Bryk, A. S. (2002). Hierarchical linear models: Applications and data analysis methods (Vol. 1). Sage. 
Rothman, N. B., \& Magee, J. C. (2016). Affective expressions in groups and inferences about members' relational well-being: The effects of socially engaging and disengaging emotions. Cognition and Emotion, 30(1), 150-166.

Santiago, P. N., Wilk, J. E., Milliken, C. S., Castro, C. A., Engel, C. C., \& Hoge, C. W. (2010). Screening for alcohol misuse and alcohol-related behaviors among combat veterans. Psychiatric Services, 61(6), 575-581.

Sayer, N. a, Noorbaloochi, S., Frazier, P., Carlson, K., Gravely, A., \& Murdoch, M. (2010). Reintegration problems and treatment interests among Iraq and Afghanistan combat veterans receiving VA medical care. Psychiatric Services, 61(6), 589-597. https://doi.org/10.1176/ps.2010.61.6.589

Scherbaum, C. A., \& Ferreter, J. M. (2009). Estimating statistical power and required sample sizes for organizational research using multilevel modeling. Organizational Research Methods, 12(2), 347-367.

Shorey, R. C., McNulty, J. K., Moore, T. M., \& Stuart, G. L. (2017). Trait Anger and Partner-Specific Anger Management Moderate the Temporal Association Between Alcohol Use and Dating Violence. Journal of Studies on Alcohol and Drugs, 78(2), 313-318. https://doi.org/10.15288/jsad.2017.78.313

Shorey, R. C., Stuart, G. L., Moore, T. M., \& McNulty, J. K. (2014). The temporal relationship between alcohol, marijuana, angry affect, and dating violence perpetration: A daily diary study with female college students. Psychology of Addictive Behaviors, 28(2), 516. 
Shmueli, D., \& Prochaska, J. J. (2012). A test of positive affect induction for countering self-control depletion in cigarette smokers. Psychology of addictive behaviors, $26(1), 157$.

Smith, T. W., Glazer, K., Ruiz, J. M., \& Gallo, L. C. (2004). Hostility, anger, aggressiveness, and coronary heart disease: An interpersonal perspective on personality, emotion, and health. Journal of personality, 72(6), 1217-1270.

Soldatos CR, Allaert FA, Ohta T, Dikeos DG. (2005) How do individuals sleep around the world? Results from a single-day survey in ten countries. Sleep Med 6:5-13.

Smyth, J. M., Hockemeyer, J. R., \& Tulloch, H. (2008). Expressive writing and posttraumatic stress disorder: Effects on trauma symptoms, mood states, and cortisol reactivity. British Journal of Health Psychology, 13(1), 85-93.

Spector, P. E., Fox, S., \& Domagalski, T. (2006). Emotions, violence and counterproductive work behavior. Handbook of workplace violence, 29-46.

Spielberger, C. D. (1988). State-trait anger expression inventory research edition. Professional manual. Odessa, FL: Psychological Assessment Resources.

Spielberger, C. D., Jacobs, G., Russell, S., \& Crane, R. S. (1983). Assessment of anger: The state-trait anger scale. Advances in personality assessment, 2, 159-187.

Steele, N. M., \& Fogarty, G. J. (2017). Screening for Anger and Sleep Difficulties. Military Medicine, 182(3), e1628-e1633. https://doi.org/10.7205/MILMED-D$16-00187$ 
Stockwell, T., Donath, S., Cooper-Stanbury, M., Chikritzhs, T., Catalano, P., \& Mateo, C. (2004). Under-reporting of alcohol consumption in household surveys: a comparison of quantity-frequency, graduated-frequency and recent recall. Addiction, 99(8), 1024-1033.

Tabachnick, B., \& Fidell, L. (1996). Using multivariate statistics (3rd ed.). New York: HarperCollins College.

Tanielian, T., Batka, C., \& Meredith, L. S. (2017). The Changing Landscape for Veterans' Mental Health Care. Santa Monica, CA: RAND Corporation. https://www.rand.org/pubs/research briefs/RB9981z2.html.

Tangney, J. P., Baumeister, R. F., \& Boone, A. L. (2004). High self-control predicts good adjustment, less pathology, better grades, and interpersonal success. Journal of personality, 72(2), 271-324.

Tavris, C. (1989). Anger, the Misunderstood Emotion. New York, NY, Simon and Schuster.

UCLA Statistical Consulting Group. (UCLA, n.d.). Retrieved from https://stats.idre.ucla.edu/mplus/dae/zero-inflated-negative-binomial-regression/

Veenstra, L., Bushman, B. J., \& Koole, S. L. (2018). The facts on the furious: a brief review of the psychology of trait anger. Current Opinion in Psychology, 19, 98103. https://doi.org/10.1016/i.copsyc.2017.03.014.

Vohs, K. D., \& Faber, R. J. (2007). Spent resources: Self-regulatory resource availability affects impulse buying. Journal of consumer research, 33(4), 537-547. 
Watson, D., \& Clark, L. A. (1994). The PANAS-X: Manual for the Positive and Negative Affect Schedule-Expanded Form. Ames: The University of Iowa.

Wilk, J. E., Quartana, P. J., Clarke-Walper, K., Kok, B. C., \& Riviere, L. A. (2015).

Aggression in US soldiers post-deployment: Associations with combat exposure and PTSD and the moderating role of trait anger. Aggressive Behavior, 41(6), 556-565. https://doi.org/10.1002/ab.21595

Worthen, M., Rathod, S. D., Cohen, G., Sampson, L., Ursano, R., Gifford, R., ... Ahern, J. (2014). Anger problems and posttraumatic stress disorder in male and female National Guard and Reserve Service members. Journal of Psychiatric Research, 55(1), 52-58. https://doi.org/10.1016/j.jpsychires.2014.04.004

Zeileis, A., Kleiber, C., \& Jackman, S. (2008). Regression models for count data in R. Journal of Statistical Software, 27(8), 1-25. 


\section{APPENDIX}

\section{Survey Items}

\section{Demographics}

What is your age?

What is your gender?

Are you of Hispanic, Latino, or Spanish Origin?

What is your race/ethnicity? (Select all that apply)

American Indian or Alaska Native

Asian

Black or African American

Native Hawaiian or Pacific Islander

Non-Hispanic White

Other

What is the highest degree or level of school you have completed?

What is your current marital status?

Single

In a committed relationship (not cohabitating)

Cohabitating (not married)

Married

Divorced

Separated

Widowed

Civil commitment or union

\section{Military Characteristics}

What best describes your current military status?

Active

Separated

How long have you been or were you in the military?

What is your present or most recent rank/pay grade?

Number of times deployed including foreign and domestic post 9/11? 


\section{Trait Anger}

Dimensions of Anger Reactions Scale (DAR; Forbes et al., 2004)

Instructions: Please indicate the degree to which each statement describes your feelings or behavior.

$1=$ Not at all, $8=$ Exactly so

1. I often find myself getting angry at people or situations.

2. When I get angry, I get really mad.

3. When I get angry, I stay angry.

4. When I get angry at someone, I want to clobber the person.

5. My anger interferes with my ability to get my work done.

6. My anger prevents me from getting along with people as well as I'd like to.

7. My anger has a bad effect on my health.

Daily Anger

Positive and Negative Affect Schedule (PANAS-X, Watson \& Clark, 1994)

Instructions: This is a list of feelings and emotions. Indicate to what extent you feel this way RIGHT NOW

$1=$ Not at all, $5=$ Extremely

\section{Angry}

\section{Daily Drink Status/Quantity}

How many drinks containing alcohol did you consume?

0

1

2

3

4

5

6

7

8

More than 8 